\title{
LHomme
}

L'HOMME Revue française d'anthropologie

$190 \mid 2009$

Varia

\section{François Robinne, Prêtres et chamanes}

\section{Bernard Formoso}

\section{OpenEdition}

Journals

Édition électronique

URL : http://journals.openedition.org/lhomme/28745

DOl : 10.4000//homme.28745

ISSN : 1953-8103

\section{Éditeur}

Éditions de l'EHESS

\section{Édition imprimée}

Date de publication : 1 janvier 2009

Pagination : 240-242

ISSN : 0439-4216

\section{Référence électronique}

Bernard Formoso, « François Robinne, Prêtres et chamanes », L'Homme [En ligne], 190 | 2009, mis en

ligne le 03 janvier 2017, consulté le 24 septembre 2020. URL : http://journals.openedition.org/ Ihomme/28745; DOI : https://doi.org/10.4000//homme.28745

Ce document a été généré automatiquement le 24 septembre 2020.

(c) École des hautes études en sciences sociales 


\title{
François Robinne, Prêtres et chamanes
}

\author{
Bernard Formoso
}

\section{RÉFÉRENCE}

François RoBINNE, Prêtres et chamanes. Métamorphoses des Kachin de Birmanie, Paris, L'Harmattan, 2007, 312 p., bibl., index, gloss., ill. (« Recherches asiatiques »)

PAR SA PROBLÉMATIQUE, tournée vers l'analyse des relations interethniques en contexte birman, ce livre se place dans le droit fil de l'étude que l'auteur consacra il y a quelques années aux populations du lac Inle (États shan) ${ }^{1}$ et, sur le plan de l'objet choisi, dans le prolongement d'une riche littérature ethnographique échelonnée sur près d'un siècle et relative aux Kachin ${ }^{2}$. Tout en reconnaissant à Edmund Leach le mérite d'avoir été le premier à souligner l'importance du relationnel externe dans les constructions identitaires, François Robinne n'en corrige pas moins sur plusieurs points l'ethnographie du célèbre ethnologue britannique relative aux Kachin. En ce sens, il reprend certaines des critiques énoncées par Maran La Raw ou Wang Zhusheng. Ainsi les termes gumsa et gumlao ne désignent pas des types d'organisation - l'un hiérarchique, l'autre égalitaire -, mais bien plutôt des modes de basculement d'une structure politique vers une autre. D'après les catégories vernaculaires, ces structures politiques sont nommées gumchying gumsa et gumrawng gumsa, avec gumyu comme terme intermédiaire. Toutes sont hiérarchiques à des degrés variables. De plus, selon François Robinne (p. 213), le rapprochement que tente Leach entre les principautés shan et les chefferies kachin, usage commun du terme mung à l'appui, n'est guère pertinent car l'autorité des chefs kachin ne débordait pas le cadre d'un village contrairement aux mung shan. Autre remarque de l'auteur, Leach minimisa à tort le poids des liens de solidarité indéfectibles existant entre aristocrates et roturiers que sous-tendait la relation donneurs - preneurs d'épouses (mayu-dama), dans le cadre de l'échange généralisé. En dépit de la grande hétérogénéité culturelle de l'ensemble kachin, le principal facteur d'intégration de ses multiples composantes serait cette 
relation mayu-dama, vraisemblablement empruntée aux Jinghpaw. L'auteur soutient qu'en contexte birman et du fait de l'hégémonisme politique local des Jinghpaw, les Maru, Atsi, Rawang, Lawngwaw et Lisu auraient de longue date partagé avec les premiers leur structure d'échange matrimoniale qui offrirait, selon la formule de Leach, les termes d'un langage rituel commun. Des correspondances partielles relevées entre la nomenclature clanique de ces différents groupes et, sur cette base, l'extension interethnique des règles d'exogamie soutiennent l'argument. Cependant, en dépit du caractère très suggestif de la proposition, l'auteur est le premier à admettre qu'elle s'appuie sur un faible nombre de témoignages et que la réelle portée sociologique des échanges mayu-dama entre groupes reste à démontrer.

L'ouvrage n'apporte pas seulement des nuances et de nouveaux éclairages à l'ethnographie des structures sociales et politiques kachin. Sur la base de recherches conduites entre 2000 et 2004 au cœur de l'État kachin, notamment dans la ville de Myitkyina et ses environs, il entend aussi rendre compte de l'évolution contemporaine des constructions identitaires de cet ensemble multi-ethnique et des tensions sociologiques et idéologiques qu'elles expriment. En effet, l'ethnographie la plus fournie concernant les Kachin de Birmanie date de l'époque coloniale (Hanson, Gilhodes, Leach); or les groupes rassemblés sous ce label ont vécu des changements considérables entre 1947 et 1994, longue période de confrontation entre l'État birman et la Kachin Independant Army (kio) d'obédience chrétienne. Selon l'auteur, un levier important de ces changements fut l'abrogation en 1963 des chefferies par la kio, qui entendait ainsi réduire les risques de dissension interne. Cette abrogation était en fait l'aboutissement d'un processus d'éclatement des chefferies villageoises et des lignages amorcé dès le XIX ${ }^{e}$ siècle. Ce processus a part liée avec la relocalisation de nombreux villages dans les basses terres qu'encouragèrent les missionnaires chrétiens et qu'imposèrent au cours des années de guerre les militaires birmans. Au lieu de faciliter les échanges avec les Birmans, ces mouvements se soldèrent par un phénomène de ghettoïsation orchestré à la fois par l'État et par les missions chrétiennes qui évitaient la mixité avec les bouddhistes. Malgré ce phénomène, l'auteur note que la birmanisation des jeunes générations, résultant de leur migration vers les villes, contribue à éroder le système d'échanges mayu-dama et les rapports entre membres du même lignage hors de la proche parentèle. Les prêtres chrétiens ont aussi largement contribué à la destruction des objets de culte animistes tout en se réappropriant des thèmes majeurs de la mythologie.

3 La « métamorphose kachin » qui donne son titre à l'ouvrage tient justement à l'impact croissant de la christianisation. L'auteur y consacre de longs développements (chap. III à V). Quoique aujourd'hui plus de $80 \%$ des Kachin soient convertis, certains sont baptistes, d'autres évangélistes, d'autres catholiques et certains même de culte anglican. Selon François Robinne, une telle hétérogénéité confessionnelle empêche le mouvement pan-kachin d'émerger en tant que force unifiée, capable de résister au processus de birmanisation. En effet, les réseaux de portée limitée que le christianisme tisse ne parviennent pas à relayer efficacement ceux construits par l'entremise de l'échange généralisé et qui sont désormais relégués au second plan.

4 Le langage rituel qui, selon l'auteur, fonde l'appartenance à l'ensemble kachin s'étend, à partir de la plateforme commune que constitue le système d'échange mayu-dama, aux registres de la mythologie et de l'activité cultuelle. Les chamanes ont joué et continuent à jouer un grand rôle en ce sens de par la fonction incontournable qu'ils assument pour 
le transfert des défunts dans l'au-delà et du fait de l'implication rituelle des donneurs et preneurs d'épouses dans le processus. Le quatrième chapitre de l'ouvrage, le plus détaillé sur le plan ethnographique, rend très bien compte des liens de sens que les chrétiens s'efforcent de maintenir avec un substrat religieux plus ancien, via diverses manipulations symboliques. Ainsi, dans le même temps que les églises chrétiennes ont tendance à se construire en alternative du polythéisme bouddhique birman, elles ne peuvent faire autrement que d'adopter une approche syncrétique qui perpétue les éléments fondamentaux de la cosmologie, de la mythologie et des panthéons divins antérieurs.

Dans ce livre, François Robinne défend l'idée que l'ensemble kachin doit être appréhendé sous l'angle de la dynamique des réseaux plutôt que dans l'optique des catégories ethniques et des particularismes qui leur sont associés. Dégager les différences entre groupes en contact serait en effet moins important que de mettre en avant ce qu'ils partagent. Ce parti pris méthodologique est justifié par la grande complexité de ce conglomérat multi-ethnique, composé de plus de six groupes clairement différenciés et, en leur sein, d'un nombre de clans et de lignages en constante augmentation. On regrette toutefois que François Robinne n'ait pu cerner la portée sociologique des institutions et réseaux auxquels il prête un pouvoir fédérateur et qu'il fasse peu état des interprétations, sans doute discordantes, par lesquelles les représentants des divers groupes conçoivent leur position et leur rôle dans l'édifice commun. La part respective des clivages confessionnels, ethniques ou idéologiques dans les tensions internes au mouvement pan-kachin ne ressort pas clairement de l'analyse et l'on ne dispose pas non plus de clés pour saisir comment ce mouvement est perçu de l'extérieur, notamment par les représentants de l'État birman. Compte tenu cependant des sévères restrictions que les autorités locales opposent aux investigations ethnographiques, cette étude n'en offre pas moins un utile aperçu des évolutions contemporaines d'un complexe multi-ethnique qui a longtemps été l'un des points focaux de la réflexion anthropologique.

\section{NOTES}

1. Cf. François Robinne, Fils et maîtres du Lac. Relations interethniques dans l'État Shan de Birmanie, Paris, CNRS Éd. - Éd. de la MSH, 2000 («Chemins de l'ethnologie »).

2. Ola Hanson, The Kachins. Their Customs and Traditions, Rangoon, 1919; Charles Gilhodes, The Kachins. Religion and Customs, Calcutta, Catholic Orphan Press, 1922 ; Edmund Leach, Political Systems of Highland Burma, Boston, Beacon Press, 1964 [1 $1^{\text {re }}$ éd. : 1954]; Maran La Raw, « Towards a Basis for Understanding the Minorities in Burma: The Kachin Example », in Peter Kunstadter, ed., Southeast Asian Tribes. Minorities and Nations, Princeton, Princeton University Press, 1967 ; Wang Zhusheng, The Jingpo. Kachin of the Yunnan Plateau, Tempe, Program for Southeast Asian Studies, 1997. 


\section{AUTEURS}

\section{BERNARD FORMOSO}

Université Paris-X, Département d'ethnologie, Nanterre.

bernard.formoso@mae.u-paris10.fr 